\title{
COMPACTNESS CRITERIA FOR RIEMANNIAN MANIFOLDS
}

\author{
GREGORY J. GALLOWAY
}

\begin{abstract}
Ambrose, Calabi and others have obtained Ricci curvature conditions (weaker than Myers' condition) which ensure the compactness of a complete Riemannian manifold. Using standard index form techniques we relate the problem of finding such Ricci curvature criteria to that of establishing the conjugacy of the scalar Jacobi equation. Using this relationship we obtain a Ricci curvature condition for compactness which is weaker than that of Ambrose and, in fact, which is best among a certain class of conditions.
\end{abstract}

One of the most well-known results relating the curvature and topology of a complete Riemannian manifold $M$ is the classical theorem of Myers [8] which states that if the Ricci curvature with respect to unit vectors on $M$ has a positive lower bound then $M$ is compact. (Myers also gives a diameter estimate in terms of this bound.) In 1957 Ambrose [1] published an interesting generalization of Myers' theorem. He proved that if there is a point $q M$ in such that along each geodesic $\gamma$ : $[0, \infty) \rightarrow M$ emanating from $q$ (and parameterized by arc length $t$ ) the Ricci curvature satisfies

$$
\int_{0}^{\infty} \operatorname{Ric}\left(\frac{d \gamma}{d t}, \frac{d \gamma}{d t}\right) d t=+\infty
$$

then $M$ is compact. One of the important features of this result is that the Ricci curvature is not required to be everywhere nonnegative. The author, together with T. Frankel, has applied Ambrose's theorem to certain problems in general relativity (see [4]).

In this paper we present a general technique for establishing compactness criteria for complete Riemannian manifolds. As an application of this technique we obtain a generalization of Ambrose's theorem, which, with respect to a certain class of curvature conditions, is best. This generalization can be used to improve some of the results in [4]. (See, especially, Theorem 5 and Corollary 6 of that paper.)

We take a moment to introduce some notation and terminology. Throughout, let $M$ denote a smooth complete Riemannian manifold of dimension $n>2$. Let $\langle$, $\rangle$ be the Riemannian metric on $M$ and let $\nabla$ be the associated Levi-Civita connection. If $t \rightarrow \gamma(t)$ is a curve in $M$, let $D / d t$ be the covariant derivative operator on vector fields along $\gamma$ induced by the connection $\nabla$. For vector fields $X$ and $Y$ let $R(X, Y)$ be the Riemann curvature transformation, i.e.

$$
R(X, Y) Z=\nabla_{X} \nabla_{Y} Z-\nabla_{Y} \nabla_{X} Z-\nabla_{[X, Y]} Z
$$

Received by the editors October 3, 1980.

1980 Mathematics Subject Classification. Primary 53C20.

() 1982 American Mathematical Society 0002-9939/82/0000-0025/\$02.25 
Let $K(X \wedge Y)$ be the sectional curvature of the plane spanned by the vectors $X$ and $Y$. In terms of the Riemann curvature,

$$
K(X \wedge Y)=\langle R(X, Y) Y, X\rangle /\|X \wedge Y\|^{2},
$$

where $\|X \wedge Y\|^{2}$ is the square of the area of the parallelogram spanned by $X$ and $Y$, i.e. $\|X \wedge Y\|^{2}=\langle X, X\rangle\langle Y, Y\rangle-\langle X, Y\rangle^{2}$.

The differential equation of Jacobi type,

$$
x^{\prime \prime}+r(t) x=0
$$

where $r(t)$ is continuous on an interval $I$, is said to be conjugate on $I$ if there exists a nontrivial solution $\phi(t)$ on $I$ which vanishes for at least two values $t_{1}, t_{2}$ in $I$.

The technique we shall use for establishing compactness criteria is provided by the following lemma (compare [2]).

LEMMA 1. Suppose there is a point $q$ in $M$ such that along each geodesic $\gamma:[0, \infty) \rightarrow M$ emanating from $q$ (and parameterized by arc length $t$ ) the differential equation (1), with $r(t)=\operatorname{Ric}(d \gamma / d t, d \gamma / d t) /(n-1)$, is conjugate on $[0, \infty)$. Then $M$ is compact.

Proof. The proof we give combines a lemma of Ambrose together with some standard Morse index theory techniques (see [5, 8]). In [1], Ambrose shows that if there is a point $q$ in $M$ such that every geodesic $\gamma$ emanating from $q$ contains a point conjugate to $q$ along $\gamma$ then $M$ is bounded and, hence, compact. Thus, it suffices to establish the existence of a point conjugate to $q$ along each geodesic $\gamma:[0, \infty) \rightarrow M$ emanating from $q$.

Since by assumption (1) is conjugate on $[0, \infty)$ there exists a nontrivial solution $\phi:[0, \infty) \rightarrow \mathbf{R}$ to (1) such that $\phi\left(t_{1}\right)=\phi\left(t_{2}\right)=0$ with $0<t_{1}<t_{2}$. Define the function $f:\left[0, t_{2}\right] \rightarrow \mathbf{R}$ as follows:

$$
f(t)= \begin{cases}0, & 0<t<t_{1} \\ \phi(t), & t_{1}<t<t_{2}\end{cases}
$$

Introduce the index form $I$ on $\left.\gamma\right|_{\left[0, t_{2}\right]}$. For piecewise smooth vector fields $X$ and $Y$ along $\left.\gamma\right|_{\left[0, t_{2}\right]}$, which vanish at the end points, define (see [3]),

$$
I(X, Y)=\int_{0}^{t_{2}}\left[\left\langle\frac{D X}{d t}, \frac{D Y}{d t}\right\rangle+\left\langle R\left(X, \frac{d \gamma}{d t}\right) Y, \frac{d \gamma}{d t}\right\rangle\right] d t
$$

Let $0=\tau_{0}<\tau_{1}<\cdots<\tau_{k}=t_{2}$ be a subdivision of $\left[0, t_{2}\right]$ such that $\left.X\right|_{\left[\tau_{1}, \tau_{1+1}\right]}$ and $\left.Y\right|_{\left[\tau_{i}, \tau_{i+1}\right]}$ are smooth for each $i$. Then the right-hand side of (2) can be integrated by parts to yield,

$$
I(X, Y)=-\sum_{i=1}^{k-1}\left\langle\Delta_{\tau_{i}} \frac{D X}{d t}, Y\right\rangle-\int_{0}^{t_{2}}\left\langle\frac{D^{2} X}{d t^{2}}+R\left(X, \frac{d \gamma}{d t}\right) \frac{d \gamma}{d t}, Y\right\rangle d t
$$

where $\Delta_{\tau_{i}} D X / d t$ is the jump in $D X / d t$ at $\tau_{i}$. 
Let $\left\{e_{1}, e_{2}, \ldots, e_{n-1}\right\}$ be $n-1$ orthonormal vector fields along $\left.\gamma\right|_{\left[0, t_{2}\right]}$ orthogonal to $d \gamma / d t$. For each $i=1, \ldots, n-1$, define $X_{i}(t)=f(t) e_{i}$. Then a straightforward computation shows

$$
\left\langle\frac{D^{2} X_{i}}{d t^{2}}+R\left(X_{i}, \frac{d \gamma}{d t}\right) \frac{d \gamma}{d t}, X_{i}\right\rangle=\left(f^{\prime \prime}+K\left(e_{i} \wedge \frac{d \gamma}{d t}\right) f\right) f
$$

on each of the two subintervals $\left[0, t_{1}\right]$ and $\left[t_{1}, t_{2}\right]$ on which $f$ is smooth. Thus substitution of (4) into (3) gives

$$
I\left(X_{i}, X_{i}\right)=-\int_{0}^{t_{2}}\left[f^{\prime \prime}+K\left(e_{i} \wedge \frac{d \gamma}{d t}\right) f\right] f d t
$$

where we have used the fact that $X_{i}$ vanishes at $t=t_{1}$. Then, using the fact that $\operatorname{Ric}(d \gamma / d t, d \gamma / d t)=\sum_{i=1}^{n-1} K\left(e_{i} \wedge d \gamma / d t\right)$, we obtain upon summation,

$$
\begin{aligned}
\sum_{i=1}^{n-1} I\left(X_{i}, X_{i}\right) & =-(n-1) \int_{0}^{t_{2}}\left(f^{\prime \prime}+r(t) f\right) f d t \\
& =-(n-1) \int_{t_{1}}^{t_{2}}\left(\phi^{\prime \prime}+r(t) \phi\right) \phi d t=0
\end{aligned}
$$

since $\phi$ is a solution to (1). Therefore, for some $i, I\left(X_{i}, X_{i}\right) \leqslant 0$. However by a standard index lemma (see, for example, Lemma 1.21 in [3]) we must have $I\left(X_{i}, X_{i}\right)>0$ unless there is some point conjugate to $q$ along $\left.\gamma\right|_{\left[0, t_{2}\right]}$. This concludes the proof of the lemma.

Thus, the problem of finding conditions on the Ricci curvature to ensure the compactness of $M$ is reduced to the problem of finding sufficient conditions on $r(t)$ for which the differential equation (1) is conjugate on $[0, \infty)$. But this is an old problem in the theory of ordinary differential equations for which there exists a vast literature. (See Swanson [11] for a survey of results up to 1968.) In fact many conditions on $r(t)$ have been obtained which imply the stronger condition that (1) is oscillatory on $[0, \infty)$. (Equation (1) is oscillatory on $[0, \infty)$ if each solution to (1) on $[0, \infty)$ has arbitrarily large, and hence infinitely many, zeros.) One such condition is due to Wintner [12, 1949]: if $\int_{0}^{\infty} r(t) d t=+\infty$ then (1) is oscillatory. Thus we observe that Ambrose's theorem is an immediate consequence of Lemma 1 and Wintner's result.

However, in 1955 R. A. Moore generalized Wintner's result as follows (see [7]): if for some $\lambda, 0 \leqslant \lambda<1$,

$$
\int_{0}^{\infty} t^{\lambda} r(t) d t=+\infty
$$

then (1) is oscillatory. Roughly speaking, Moore's criterion requires that $r(t)$ behave on the average like a function having order greater than $t^{-2}$, whereas Wintner's criterion requires $r(t)$ to behave on the average like a function having order greater than or equal to $t^{-1}$. Consideration of the Euler equation $x^{\prime \prime}+$ $\frac{1}{4}(t+1)^{-2} x=0$ (with solution $x=c_{1} \sqrt{t+1}+c_{2} \sqrt{t+1} \ln (t+1)$ ) shows that Moore's result cannot be improved to include $\lambda=1$.

Moore's result in conjunction with Lemma 1 yields the following generalization of Ambrose's theorem. 
THEOREM 2. If there is a point $q$ in $M$ such that along each geodesic $\gamma:[0, \infty) \rightarrow M$ emanating from $q$ (and parameterized by arc length $t$ ) the condition

$$
\int_{0}^{\infty} t^{\lambda} \operatorname{Ric}(t) d t=+\infty
$$

holds for some $\lambda, 0 \leqslant \lambda<1$, where $\operatorname{Ric}(t)=\operatorname{Ric}(d \gamma / d t, d \gamma / d t)$, then $M$ is compact.

Note that from a slightly different point of view, Theorem 2 gives information about the rate of decay of the Ricci curvature along some geodesic ray at each point of an open (i.e. complete, noncompact) Riemannian manifold.

We now give an example to show that the curvature condition of Theorem 2 cannot be improved to allow $\lambda=1$. Let $M=\mathbf{R}^{2}$ and be furnished with the metric (given in polar coordinates $r, \theta) d s^{2}=d r^{2}+G(r) d \theta^{2}$ where $G(r)$ is given by

$$
G(r)= \begin{cases}r^{2}, & 0 \leqslant r \leqslant \frac{1}{2}, \\ g(r), & \frac{1}{2} \leqslant r \leqslant 1, \\ r, & r \geqslant 1 .\end{cases}
$$

Here, $g(r)$ is any smooth positive function whose graph matches up smoothly with the graph of $r^{2}$ at $r=\frac{1}{2}$ and the graph of $r$ at $r=1$. (The definition of $G(r)$ for $r \leqslant \frac{1}{2}$ ensures that the metric is nonsingular at the origin.) $M$, in this metric, is complete and spherically symmetric about the origin. The radial curves are geodesics and $r$ is arc length along them. In this two dimensional example the Ricci curvature reduces to Gaussian curvature $K(r)$, and for $r \geqslant 1$,

$$
K(r)=-(1 / \sqrt{G}) \partial^{2} \sqrt{G} / \partial r^{2}=1 / 4 r^{2} .
$$

Thus, $\int_{0}^{\infty} r K(r) d r=+\infty$, yet $M$ is noncompact. This example is easily generalized to higher dimensions.

If we restrict attention to manifolds having nonnegative Ricci curvature, the curvature condition of Theorem 2 can be weakened as follows.

THEOREM 3. Assume $M$ has nonnegative Ricci curvature. If there is a point $q$ in $M$ such that along each geodesic $\gamma:[0, \infty) \rightarrow M$ emanating from $q$ (and parameterized by arc length $t$ ) the inequality

$$
\int_{t_{0}}^{\infty} t^{\lambda} \operatorname{Ric}(t) d t>(n-1) \frac{(2-\lambda)^{2}}{4(1-\lambda)} \frac{1}{t_{0}^{1-\lambda}}
$$

holds for some $t_{0}>0$ and some $\lambda, 0 \leqslant \lambda<1$, then $M$ is compact.

The theorem is a consequence of Lemma 1 and a result of Nehari's (see [9, Theorem III]) which shows that if the inequality

$$
\int_{t_{0}}^{\infty} t^{\lambda} \dot{r}(t) d t>\frac{(2-\lambda)^{2}}{4(1-\lambda)} \cdot \frac{1}{t_{0}^{1-\lambda}}
$$

holds then (1) is conjugate on $[0, \infty)$. (In fact Nehari assumes $r(t)>0$, but his arguments are easily modified to allow $r(t) \geqslant 0$.) Note that the right-hand side of 
(4) can be written in the form

$$
\frac{(2-\lambda)^{2}}{4} \int_{t_{0}}^{\infty} t^{\lambda} \cdot \frac{1}{t^{2}} d t,
$$

so that, roughly speaking, Nehari's criterion allows $r(t)$ to behave on the average like $c \cdot t^{-2}$ on $\left[t_{0}, \infty\right)$ where $c>(2-\lambda)^{2} / 4$ for some $\lambda, 0<\lambda<1$.

We present one final result which emphasizes the nature of the decay of the Ricci curvature in open manifolds of nonnegative curvature. (For a related result, see [10].)

THEOREM 4. If $M$ is open then at each point $q$ of $M$ the inequality

$$
\lim _{t \rightarrow \infty} \inf t^{2} \operatorname{Ric}(t)<\frac{n-1}{4}
$$

must hold along some geodesic $\gamma:[0, \infty) \rightarrow M$ emanating from $q$ (and parameterized by arc length $t$ ).

Proof. Suppose, on the other hand, the inequality $\lim _{t \rightarrow \infty} \inf t^{2} r(t)>\frac{1}{4}$ holds along each geodesic $\gamma$ emanating from some point $q$ (where $r(t)=\operatorname{Ric}(t) /(n-1)$ ). Then according to an oscillation result of Hille and Kneser [6], (1) is oscillatory. Hence, by Lemma $1, M$ is compact, which is a contradiction.

We remark that the example following Theorem 2 shows that the bound in (5) is sharp.

\section{REFERENCES}

1. W. Ambrose, A Theorem of Myers, Duke Math. J. 24 (1957), 345-348.

2. E. Calabi, On Ricci curvature and geodesics, Duke Math. J. 34 (1967), 667-676.

3. J. Cheeger and D. G. Ebin, Comparison theorems in Riemannian geometry, American Elsevier, New York, 1975.

4. T. Frankel and G. J. Galloway, Energy density and spatial curvature in general relativity, J. Math. Phys. 22 (1981), 813-817.

5. D. Gromoll and W. Meyer, On complete open manifolds of positive Ricci curvature, Ann. of Math. 90 (1969), 75-90.

6. E. Hille, Non-oscillation theorems, Trans. Amer. Math. Soc. 64 (1948), 234-252.

7. R. A. Moore, The behavior of solutions of a linear differential equation of second order, Pacific $J$. Math. 5 (1955), 125-145.

8. S. B. Myers, Riemannian manifolds with pasitive mean curvature, Duke Math. J. 8 (1941), $401-404$.

9. Z. Nehari, Oscillation criteria for second-order linear differential equations, Trans. Amer. Math. Soc. 85 (1957), 428-445.

10. R. Schneider, Konvexe flächen mit langsam abnehmender Krümmung, Arch. Math. 23 (1972), 650-654.

11. C. A. Swanson, Comparison and ascillation theory of linear differential equations, Academic Press, New York, 1968.

12. A. Wintner, A criterion of oscillatory stability, Quart. Appl. Math. 7 (1949), 115-117.

Department of Mathematics, University of Miami, Coral Gables, Florida 33124 